\title{
Two Dimensional Path Planning with Static Polygon Obstacles Avoidance
}

\author{
Duaa Ahmed Ramadhan*1, Abdulmuttalib T. Rashid ${ }^{2}$, Osama T. Rashid ${ }^{3}$ \\ ${ }^{1}$ Electrical Engineering Department, University of Basrah, Basrah, Iraq \\ ${ }^{2}$ Electrical Engineering Department, University of Basrah, Basrah, Iraq \\ ${ }^{3}$ Computer Engineering Department, Iraq University Collage, Basrah, Iraq
}

\begin{abstract}
Correspondence
* Duaa Ahmed Ramadhan

Electrical Engineering Department,

University of Basrah, Basrah, Iraq

Email: duaa.eng.coe1992@gmail.com
\end{abstract}

\begin{abstract}
This paper presents the designing of path planning system in an environment contains a set of static polygon obstacles localized and distributed randomly by using differential drive mobile robot. In this paper the designed algorithm (two dimensional path planning algorithm) is proposed in order of investigate the path planning of mobile robot with free collision using the visibility binary tree algorithm. The suggested algorithm is compared with the virtual circles tangents algorithm in the time of arrival and the longest of the path to the target. The aim of this paper is to get an algorithm has better performance than the other algorithms and get less time of arrival and shortest path with free collision.
\end{abstract}

KEYWORDS: Mobile robot, path planning, polygon obstacle.

\section{INTRODUCTION}

Path planning concept is a way of moving robot from the initial point to the destination point with free collision and less time arrival, and shortest path [1]. It has a wide number of applications in games [2], logistics [3], and robotics [4]. Path planning of mobile robot is one of the most challenges in robotics because it is applied in different applications such as routing transportation, organizing allocation of machines in factories, controlling robots, intelligent agents [5] and also in military applications [6]. Path planning algorithms can be implemented in static, dynamic, and real time different environments, therefore it suffers from number of problems such as single agent path planning, multi agent path planning, adversarial path planning, and changes in dynamic environments that require pre-processing operation which makes path planning complex in real time [7]. There are many algorithms used for path planning system construction. Path planning algorithms classified into two types: on-line and off-line path planning. On-line path planning suitable for a partially known environment with a static target, since the robot uses its sensors to know about the environment but not all they can be solve moving target problem [8-10]. On-line path planning depends on the current environment information to select the current optimal path [11]. Off-line path planning was not suitable for the large dynamic environment and moving target problem handling because it consumes time since the complete path from start to the target is computed at the start position [12]. Off-line path planning algorithms converted to incremental algorithms in order to be more efficient $[13,14]$. Incremental algorithms suitable for a partially known environment but not solve moving target problem. In this paper, differential-drive mobile robot is used in an environment contains fixed polygon obstacles. Visibility binary search algorithm [15], Minimum bounding circle algorithm [16, 17] are used to draw tree of paths which is contacts with obstacles then choose a good path from this tree. Also use virtual circles tangents algorithm then compare between them to get algorithm with good performance.

\section{Two Dimensional Path Planning Algorithm}

In this section of research, it will design path planning system in an environment contains a set of static polygon obstacles by using differential drive mobile robot. Method of algorithm is based on obstacle avoidance by drawing paths in contact with obstacles to configure a tree of paths using visibility binary algorithm [15]. The shortest path is chosen of this tree for moving a robot to the target point.

\section{A. Compute the centriod of each obstacle}

In this section, it will represent every obstacle with a circle contains all of polygon obstacle heads and then find a center of this circle to use it for exploration if this obstacle block the way of robot or not. For this purpose, algorithm is chosen is Minimum bounding circle algorithm [16, 17], this algorithm depends on using Chan's algorithm in Fig.1 and 2. 




Fig. 1 Chan's algorithm

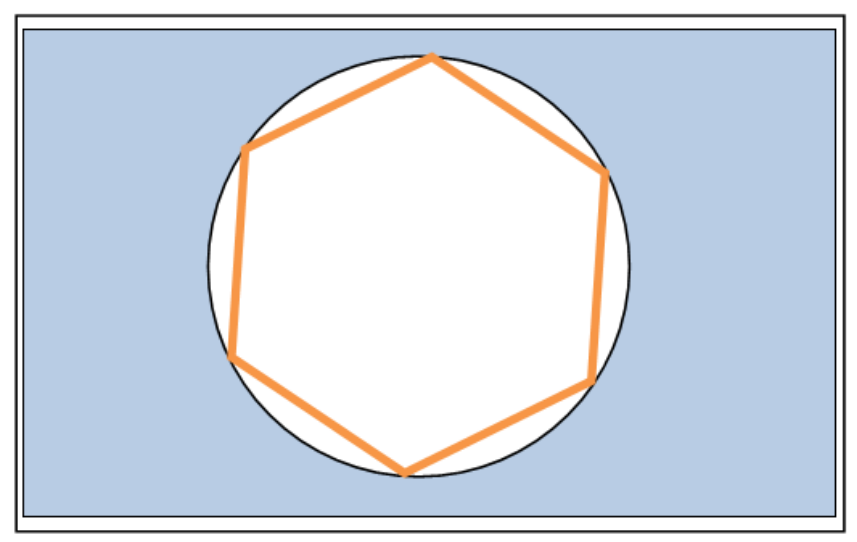

Fig. 2 Minimum bounding circle algorithm

\section{B. Binary tree paths construction algorithm}

In this section, it will construct a tree of paths for a robot according to the following steps:

Step 1: Direct path to the target: In this step, a direct path is drawing for moving robot from initial point to target point as in Fig. 3.

Step 2: Testing the collision with an obstacle: In this step, it will test an interception of one of obstacles for path of robot. At first, it will determine distance between every obstacle and path that connect between robot and target using the following relations [19] as in Fig. 4

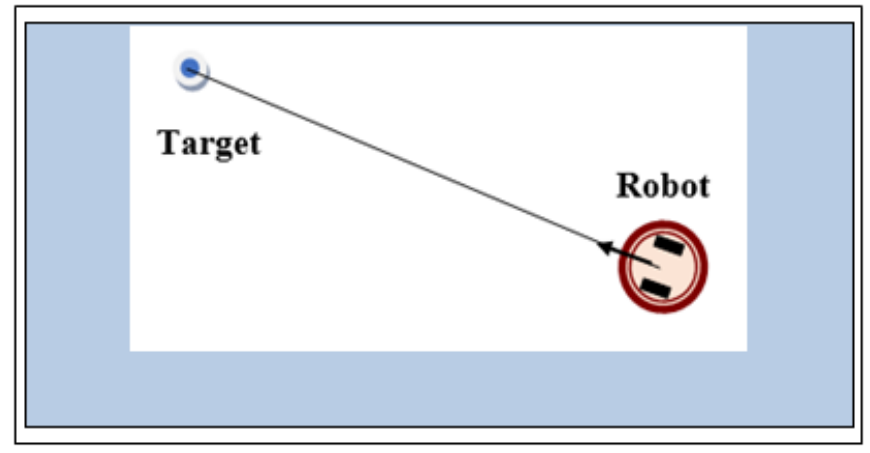

Fig. 3 Direct path

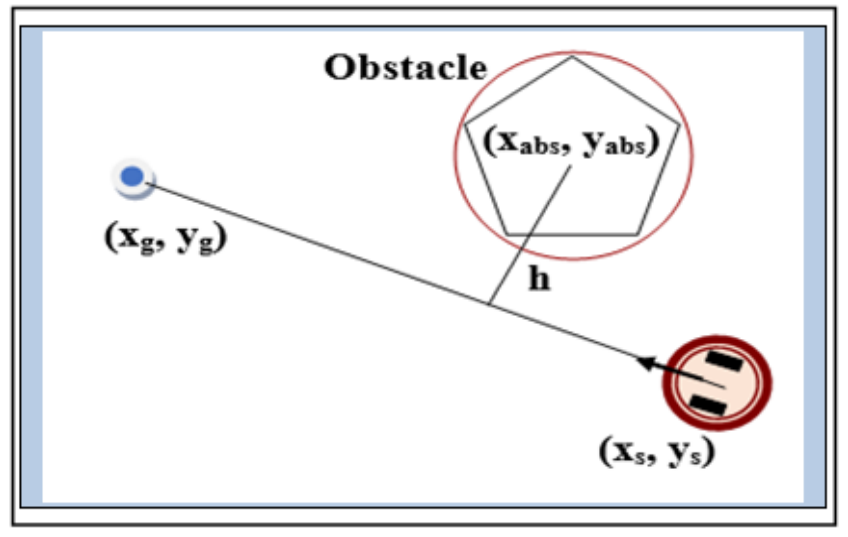

Fig. 4 Detection the collision with the obstacle

$$
\begin{aligned}
& a=(y g-y s) /(x g-x s) \\
& b=-1 \\
& c=y s-a * x s \\
& h=A b s(a * x a b s+b * y a b s+c) / \operatorname{Sqrt}(a 2+b 2))
\end{aligned}
$$

Step 3: Choose the nearest collision

In this step, it will choose obstacles that far away from the path that connect between robot and target with shortest distance from the radius of circle that surrounding obstacle. The obstacle that having minimum value of distance about robot it will be the first obstacle that intercept path of robot. The following relation determined distance between obstacle and robot:

$$
\text { Dis }=\left(\left(x_{s}-x_{a b s}\right)^{2}+\left(y_{s}-y_{a b s}\right)^{2}\right)^{0.5}
$$

Step 4: Estimate the outer and inner tangent points: In this step, it will determine the outer and inner tangent points for tangent contacts from robot to obstacle. In order to deflect direction of robot to prevent collision between it and obstacle. Estimation of tangent points by the following steps:

1. Determine distance between every head in polygon obstacle and the direct path from robot to target as in Fig. 5.

2. 2. Vertices of the polygon obstacle that having largest distance for path between robot and target represent tangent points.

Step 5: Draw the outer and inner tangent lines: In this step, it will draw lines that contacts with obstacle from robot as in Fig. 6 .

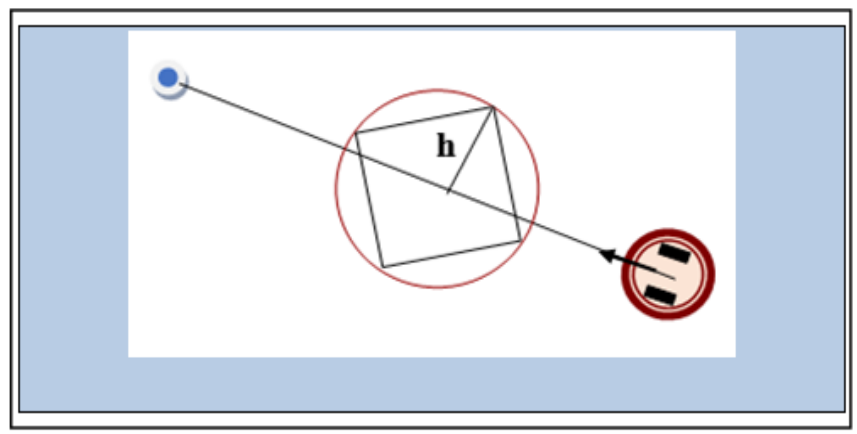

Fig. 5 Estimate the two tangent points 


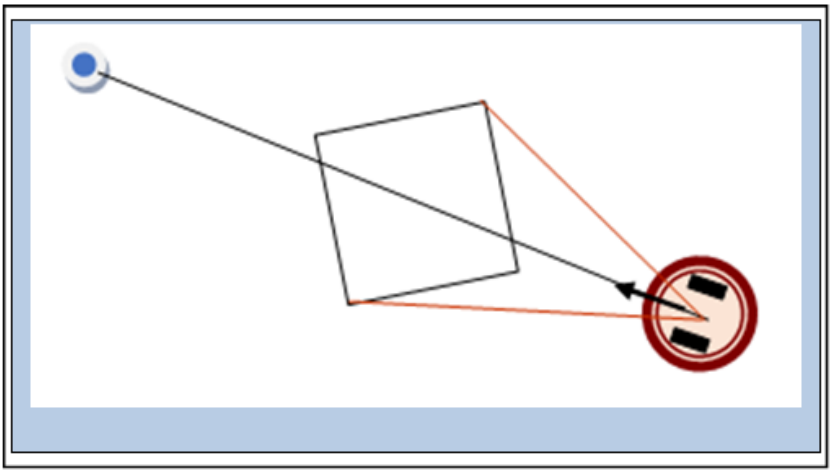

Fig. 6 The two tangent lines

Step 6: Complete the binary tree construction: Repeat the previous steps starting with every tangent point to target in order to repeat drawing tangents for obstacles that interception path of robot until the tree of tangents is complete as in Fig. 7.

\section{Optimal paths estimation}

In this part, it will choose the shortest path of a set of paths from robot and target using algorithm called visibility binary search algorithm [15] as in Fig.8.

\section{D.Low level path planning}

In this part, it will determine the way of moving robot from source to target. Robot that used in this research is differential drive mobile robot as in Fig. 9. The way of moving depends on dynamic motion for this robot.

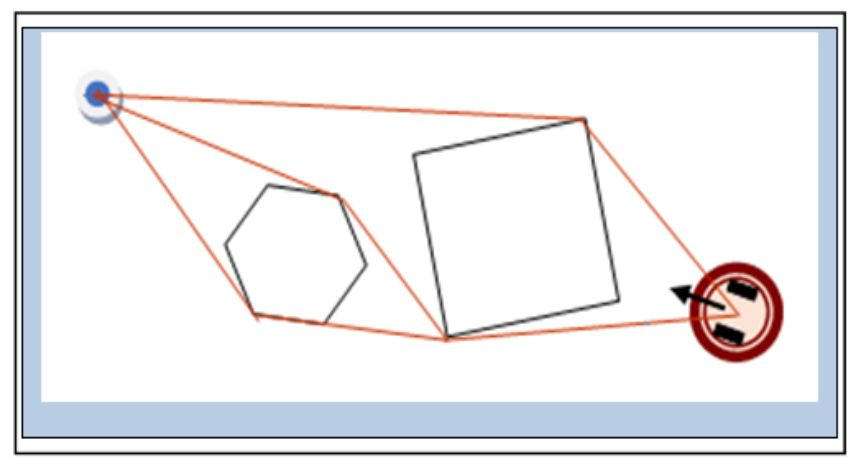

Fig. 7 Complete binary tree construction

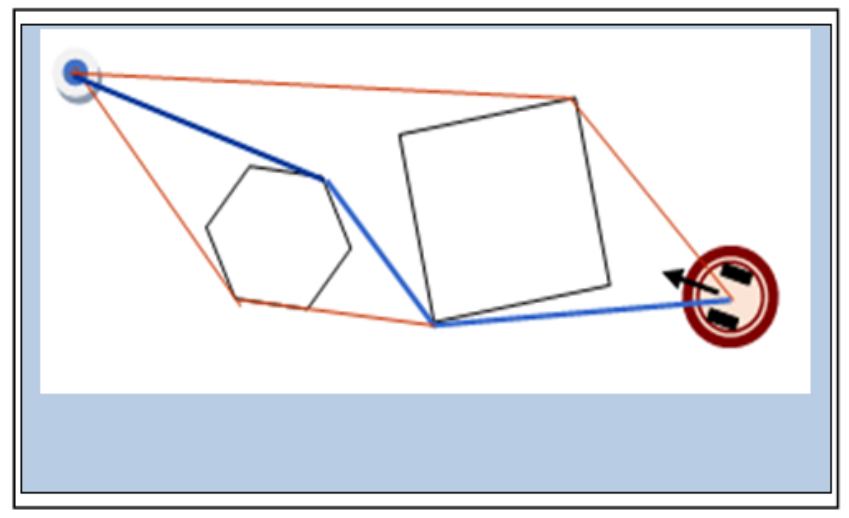

Fig.8 Optimal path from robot to the target

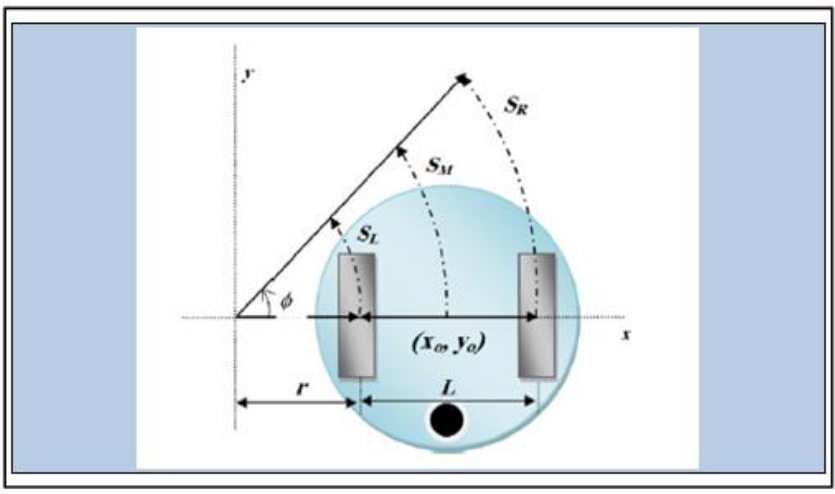

Fig. 9 Differential drive mobile robot.

Let the coordinates $\left(X_{0}, Y_{0}\right)$ represent the global position of the center of the robot in a fixed reference frame. It will consider a line is vertical to the wheel axis and goes through the point $\left(X_{0}, Y_{0}\right)$ as an orientation reference for the robot. This line make an angle $\theta$ with the positive $\mathrm{x}$ axis represents the direction of the robot. The displacement for the left and right wheels is called $S L, S R$ respectively, $r$ is the turn radius for the inner (left) wheel, $L$ represent the distance between the wheels, and $\theta$ represent the angle of the turn in radians. SM represent the displacement at the center point on the main axle. To be suitable for small robot applications where on-board computing power is limited, the approximated equations for the robot movement as follows:

$x c(t)=s \cos \theta+x_{0}$

$y c(t)=s \sin \theta+y_{0}$

Where

$s=(S R+S L) / 2$

\section{SIMULATION RESULTS}

The new algorithm (two dimensional path planning algorithm) is simulated to investigate the path planning of mobile robot using Visual basic programming language and tested in Windows environment using an Intel core i5. The different distributed of polygon shape obstacles are simulated by considering the effect of the time of arrival and the shortest distance to complete the path planning with free collision. The suggested algorithm is compared with virtual circles tangents algorithm in the time of arrival and the longest of the path to the target. Fig. 10 (a)-(f) represent the Screenshots of the simulation at different time steps for the two dimensional path planning algorithm. Fig. 11 (a)-(f) represent the Screenshots of the simulation at different time steps for the virtual circles tangent algorithm. Fig. 12 (a)-(c) represent the path planning with zero, one and two obstacles using the two dimensional path planning algorithm. Fig. 12 (d)-(f) represent the path planning with zero, one and two obstacles using the virtual circles tangent algorithm.

The main goal of this simulation is to show the relation between the number of obstacles and the time of arrival and the long the path to the goal. 


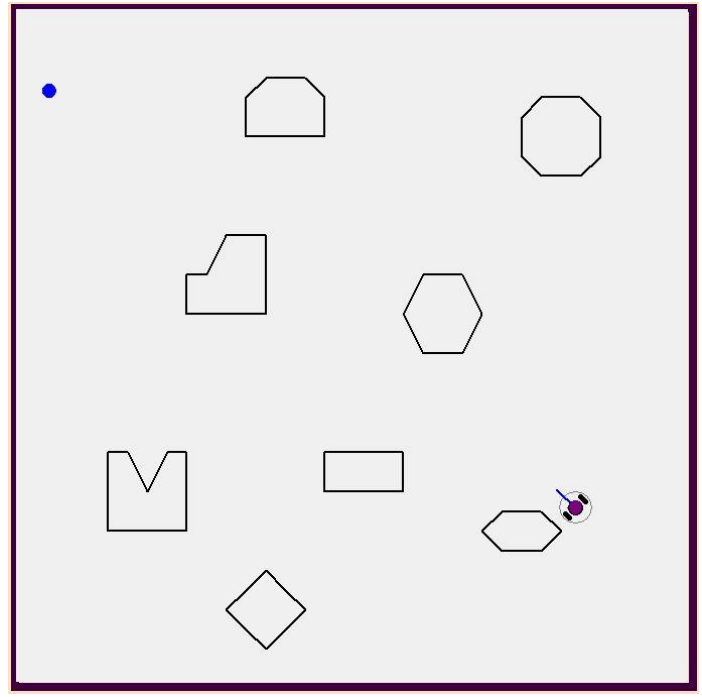

(a)

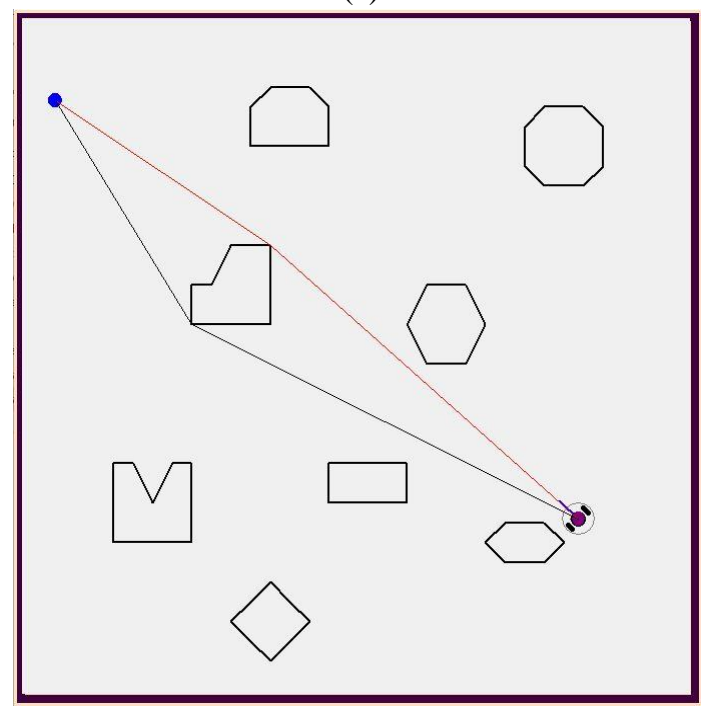

(c)

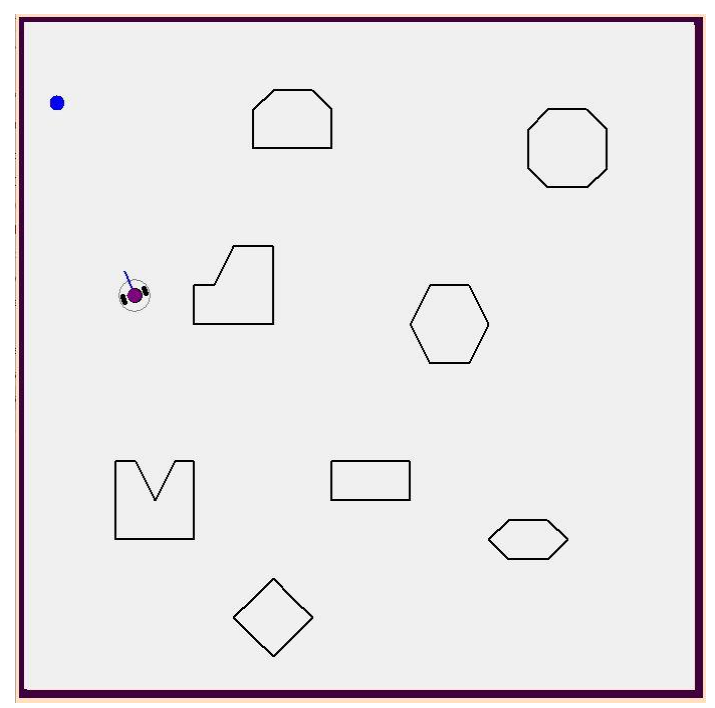

(e)

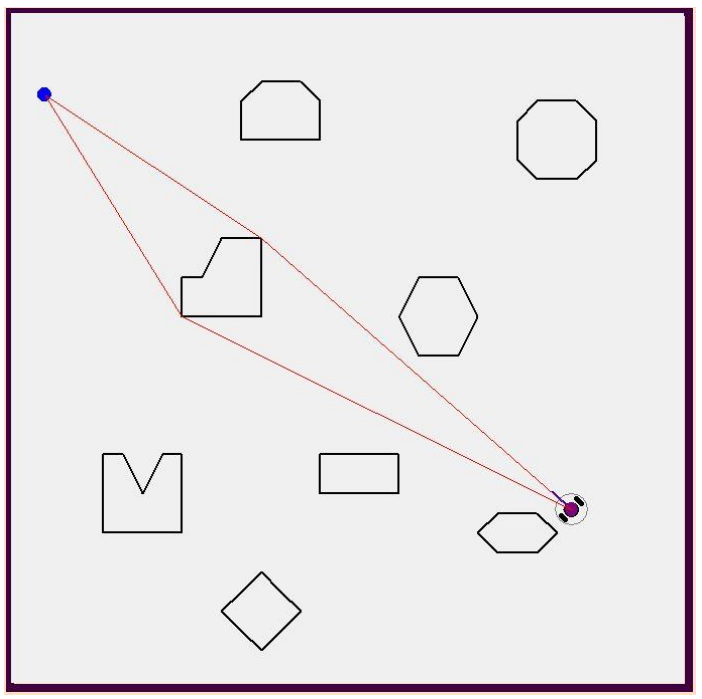

(b)

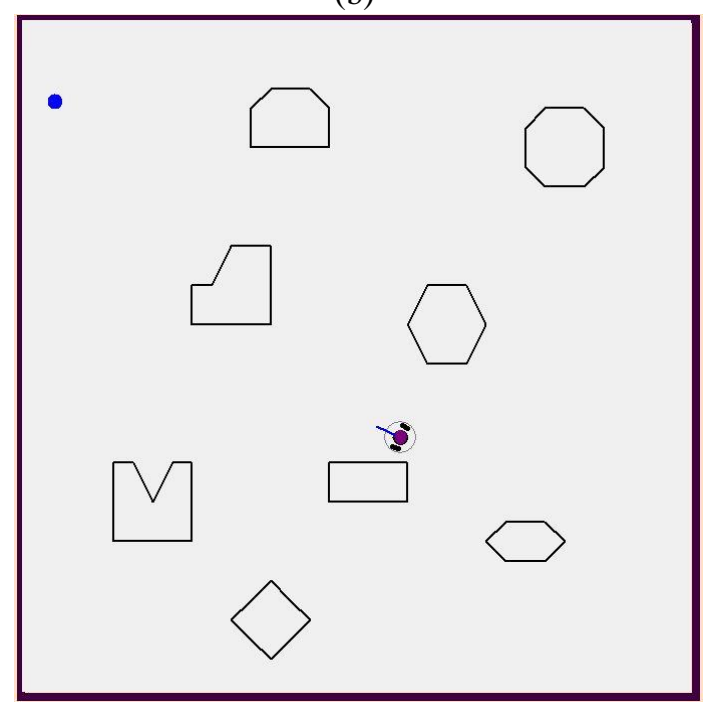

(d)

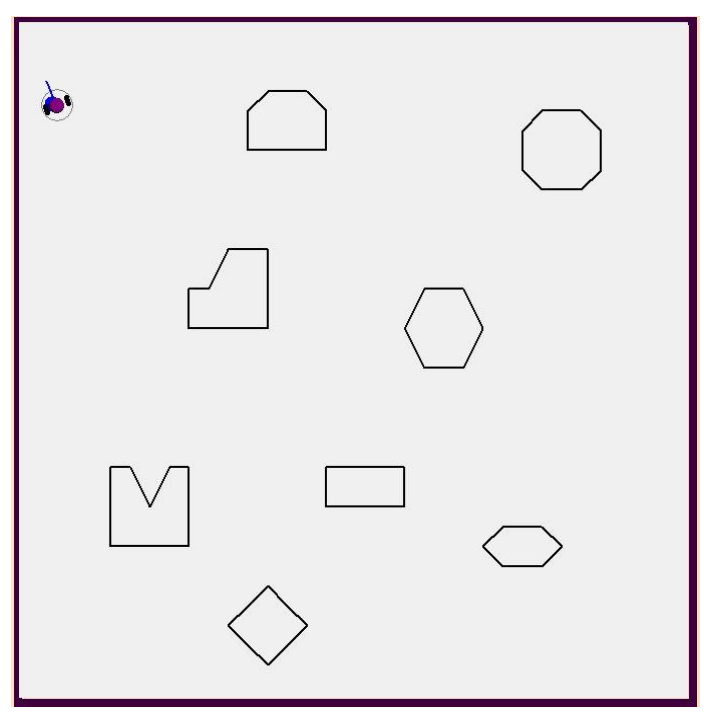

(f)

Fig.10 The two dimensional path planning algorithm. (a-f) Screenshots at different time steps trajectory planning. 


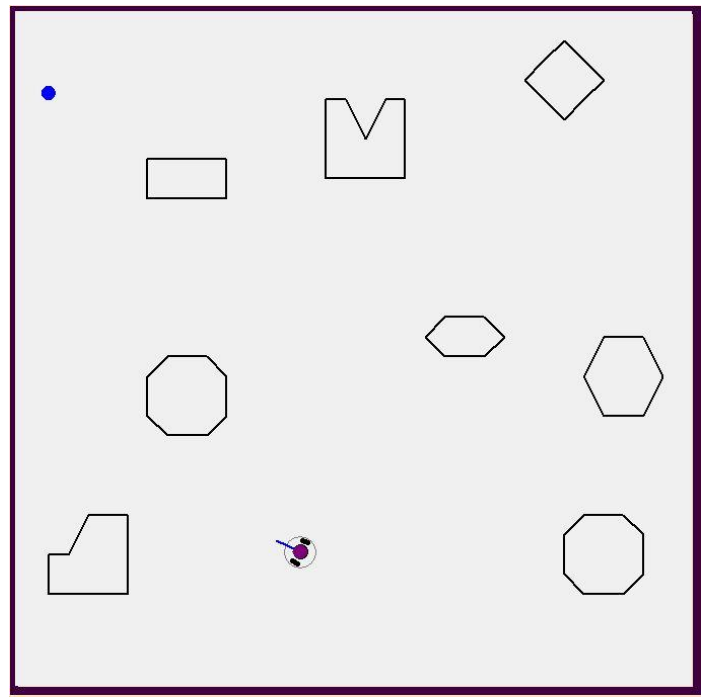

(a)

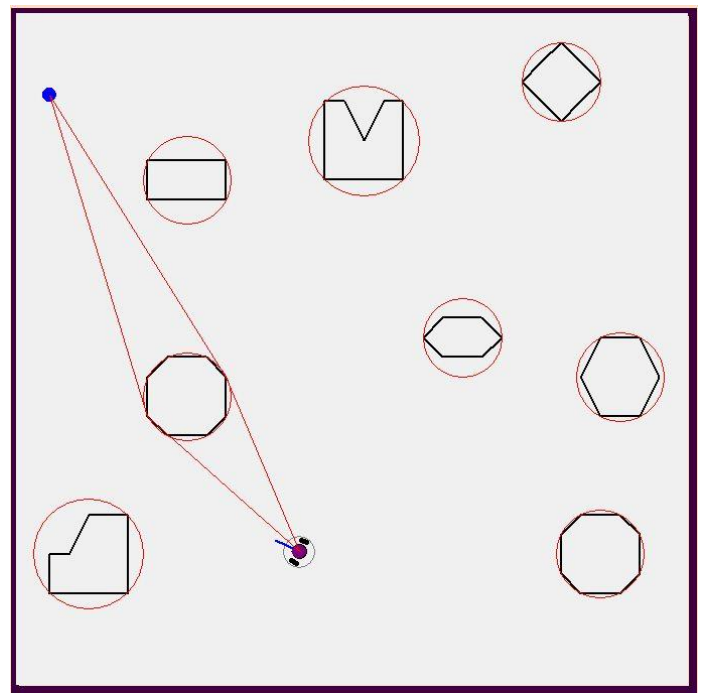

(c)

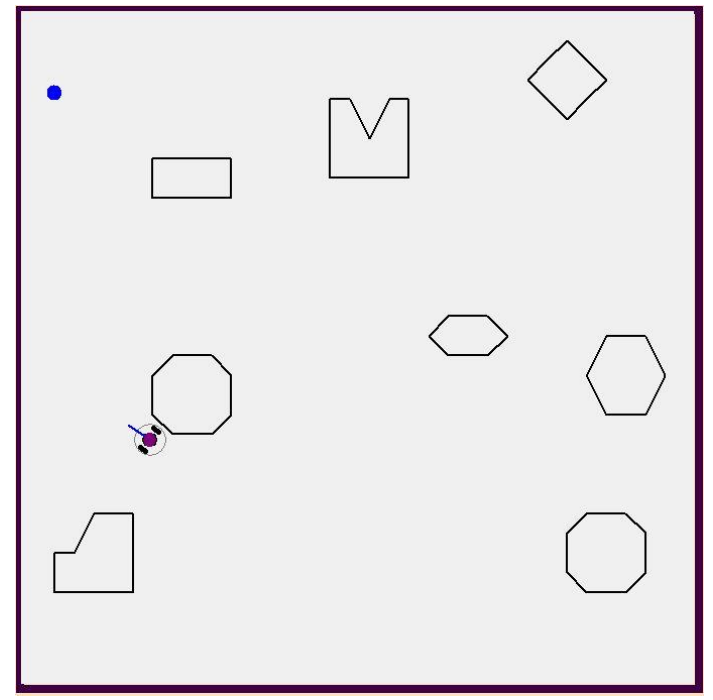

(e)



(b)



(d)

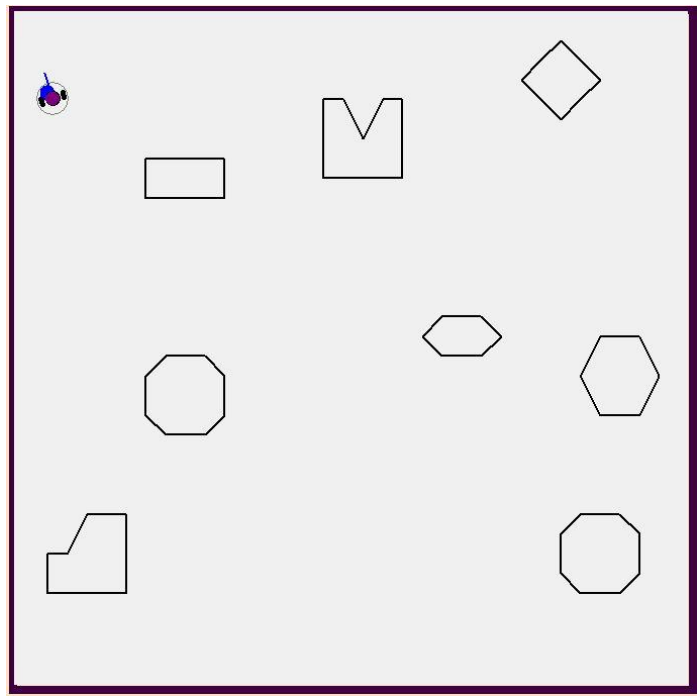

(f)

Fig.11 The virtual circles tangent algorithm. (a-f) Screenshots at different time steps trajectory planning. 


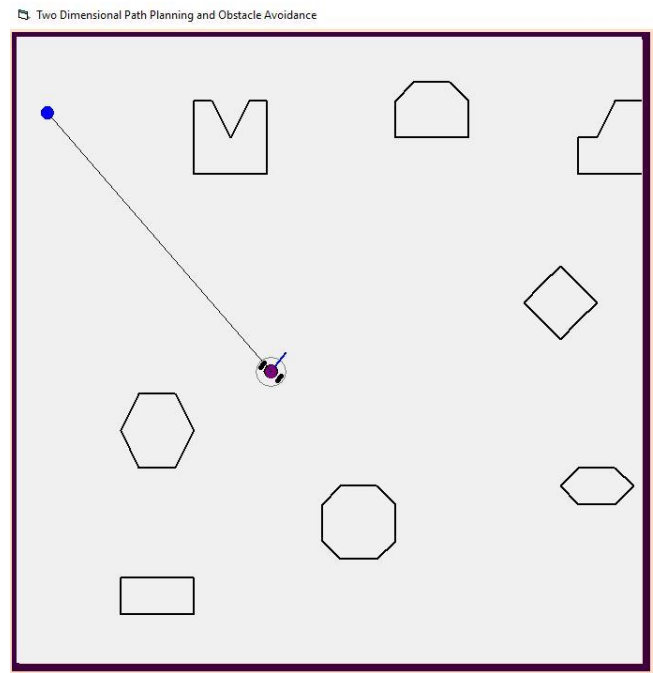

(a)

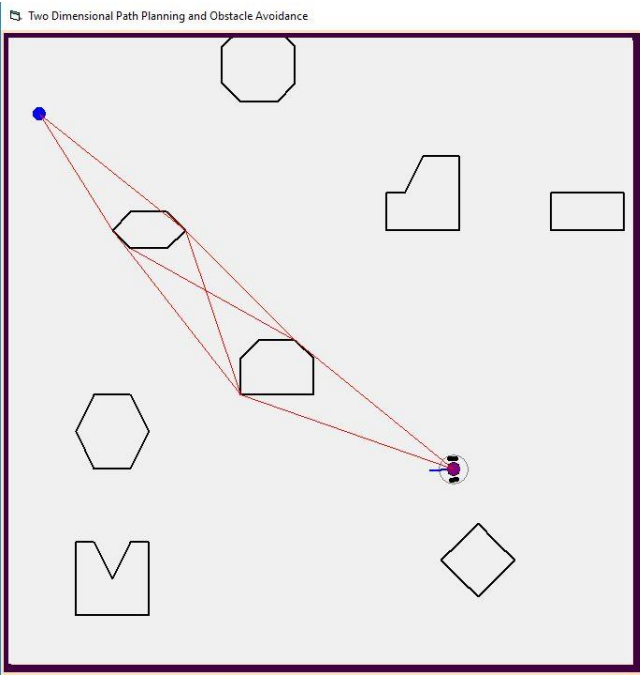

(c)

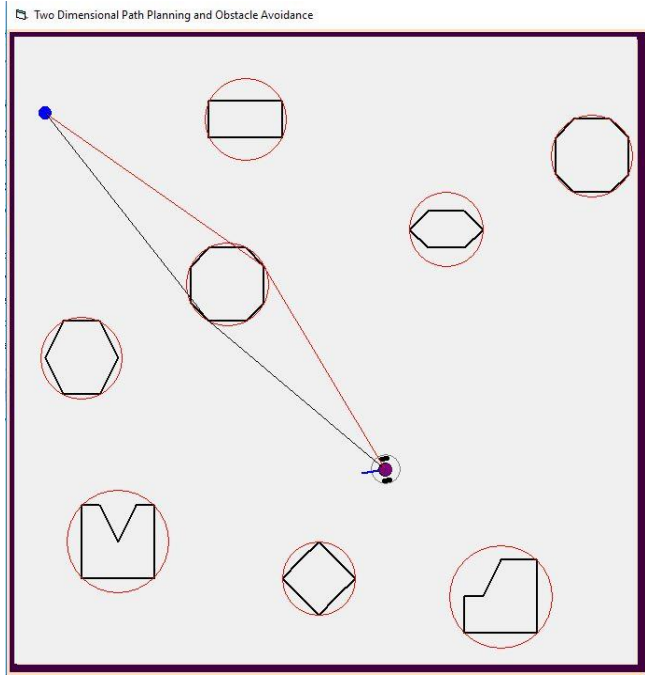

(e)



(b)



(d)

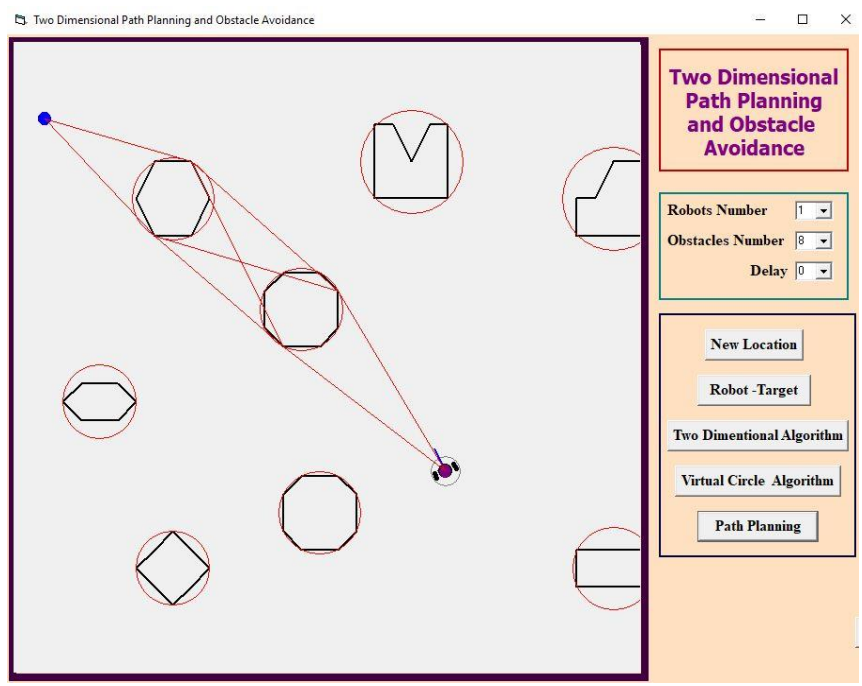

(f)

Fig.12 Path planning with different number of obstacles. (a-c) The two dimensional path planning algorithm (d-f) the virtual circles tangent algorithm. 
Fig. 13 shows the comparison between the number of obstacles and the time of arrival between the 2D path planning algorithm and the virtual circles tangent algorithm. As the number of the obstacles increase in the environment, the time of arrival increase for both algorithm but the two dimensional produced best performance than the virtual circles tangent algorithm. Fig. 14 shows the second comparison between the two dimensional path planning algorithm and the virtual circles tangent algorithm in computing the length of the path to the target. As the number of the obstacles increase in the environment, the length of the path for both algorithm is also increased. The two dimensional path planning algorithm produced best performance than the virtual circles tangent algorithm.



Fig. 13 Comparison the time of arrival for both the two dimensional path planning algorithm and the virtual circles tangent algorithm.

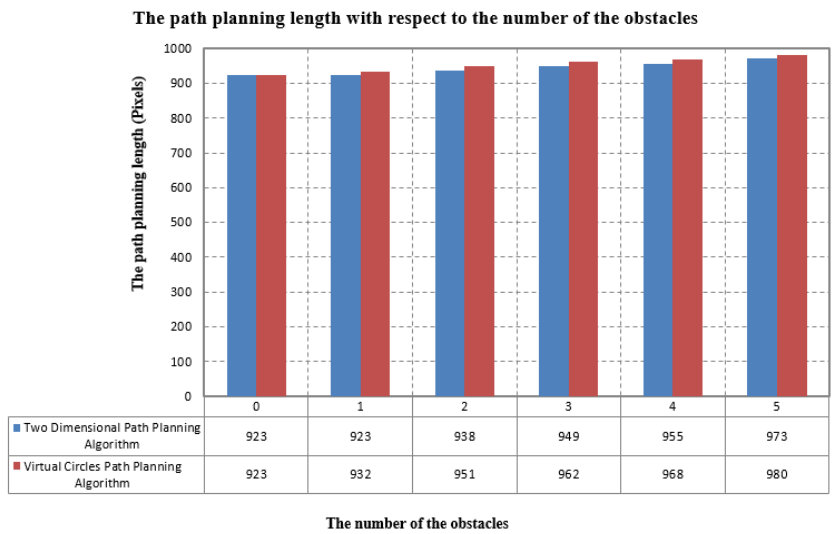

Fig. 14 Comparison the length of the path for both the two dimensional path planning algorithm and the virtual circles tangent algorithm.

\section{CONCLUSION}

In this paper, a novel two dimensional path planning algorithm in a global knowledge environment by using several numbers of obstacles localized and distributed randomly. Simulation results are implemented in an environment with a different number (0 to 8$)$ of obstacles using both the two dimensional path planning algorithm and the virtual circles tangent algorithm. The results show that both the algorithm have less time of arrival as the number of the obstacles decrease. The same results are obtained when we measure the length of the path to the target point. The suggested algorithm has better performance than the virtual circles tangent in both the time of arrival and the length of the path.

\section{CONFLICT OF INTEREST}

The authors have no conflict of relevant interest to this article.

\section{REFERENCES}

[1] Z. Y. Ibrahim, A. T. Rashid and A. F. Marhoon (2016). Prediction-Based Path Planning with Obstacle Avoidance in Dynamic Target Environment. Basrah Journal of Engineering Science, 16(2), 48-60.

[2] N.R. Sturtevant, and R. Geisberger, "Ä Comparison of High-Level Approaches for speeding Up Path finding”, As-sociation for the advancement of artificial Intelligence, pp.76-82, 2010.

[3] B. Bonet, and H. Geffner, "Planning as heuristic search", Artificial Intelligence-journal Elsevier, vol. 129, No.1-2, pp.5-33, 2001.

[4] J. Van den Berg, R.shah, A. Huang, and K. Goldberg, "ANA*: Anytime Nonparametric A*", Association for the advancement of artificial Intelligence, pp.105-111, 2011.

[5] B.coppin, Artifital Intelligent Illuminated, 2004.

[6] J.Kaur, V. K. Banga and G.Singh, "Robotic Path Planning Using the Intelligent Control", International Conference on Advances in Electrical and Electronics Engineering (ICAEE'2011).

[7] R. Graham, H. McCabe, and S. Sheridan, "Path finding in Computer Games", The IBT journal, Vol.4, pp.57-81, 2003.

[8] A.T. Rashid, A. A. Ali, M. Frasca, and L. Fortuna," An algorithm for multi-robot collision-free navigation based on shortest distance", Robotics and Autonomous Systems, Vol. 75, p.p. 119-128, 2016.

[9] Z. Y. Ibrahim, A. T. Rashid and A. F. Marhoon (2016). Path planning algorithm for mobile robot navigation in a dynamic environment based on motion prediction and tangency graph. 2017 IEEE First International Conference on Recent Trends of Engineering Science and Sustainability.

[10] C.Undeger, and F.polat," Real-Time Edge Follow: A Real-Time Path Search Approach", IEEE Transactions on Systems Man and Cybernetics Part C. October 2007.

[11] M. Shahab Alam, M. Usman Rafique, and M. Umer Khan, "Mobile Robot Path Planning in Static Environments using Particle Swarm Optimization", International journal of computer science and electronics engineering (IJCSEE) ,vol.3, pp. 253257,2015 .

[12] J.F. Canny, J.M. Malik, D.D. Edwards" Artificial Intelligence A Modern Approach", 1995.

[13] S. Koenig, and M. Likhachev," Fast Replanning for Navigation in Unknown Terrain", IEEE 
TRANSACTIONS ON ROBOTICS, VOL.21, NO.3, JUNE 2005.

[14] A.Stentz," The Focussed D* Algorithm for Real-Time Replanning”, International Joint Conference on Artificial Intelligence, August 1995.

[15] A. T. Rashid, A. A. Ali, M. Frasca, and L. Fortuna," Path planning with obstacle avoidance based on visibility binary tree algorithm", Robotics and Autonomous Systems, vol. 61, p.p1440-1449, 2013.

[16]Z. Y. Ibrahim, A. T. Rashid and A. F. Marhoon (2016). An algorithm for Path planning with polygon obstacle avoidance based on the virtual circle tangents. Iraq Journal Electrical and Electronic Engineering, 12(2), 221-234.
[17]I. S. Alfurati and A. T. Rashid (2019)," Design and Implementation an Indoor Robot Localization System Using Minimum Bounded Circle Algorithm”, 2019 8th International Conference on Modeling Simulation and Applied Optimization (ICMSAO).

[18] A. T. Rashid, A. A. Ali, M. Frasca , and L. Fortuna," Path planning and obstacle avoidance based on shortest distance algorithm", 2017 Second Al-Sadiq International Conference on Multidisciplinary in IT and Communication Science and Applications (2nd-AICMITC'17), Iraq, 2017.

[19] A. T. Rashid, A. A. Ali, M. Frasca, and L. Fortuna," Multi-robot collision-free navigation based on reciprocal orientation", Robotics and Autonomous Systems, vol. 60, p.p1221-1230, 2012. 